Pedro Manuel García-

Ceberino

Emilio Guirao-Arrabal ${ }^{2}$

Francisco Ferrer Amate $^{3}$

José Hernández-Quero²

\section{Fatal sepsis months after bladder instillations with Mycobacterium bovis in patient with SARS-CoV-2 infection}

\begin{abstract}
${ }^{1}$ Internal Medicine Unit. Hospital Universitario San Cecilio. Granada. Spain.
${ }^{2}$ Infectious Diseases Unit. Hospital Universitario San Cecilio. Granada. Spain.

${ }^{3}$ Microbiology Unit. Hospital Universitario San Cecilio. Granada. Spain.
\end{abstract}

Article history

Received: 28 June2021; Accepted: 21 August 2021; Published: 10 November 2021

\section{Sir,}

A 72-years-old man was admitted last April 2020 to our hospital with a five-days evolution of general weakness and marked fatigue. He had a history of superficial bladder cancer diagnosed four years before. Bladder cancer had been managed with fifteen instillations of intravesical infusions of bacillus Calmette-Guérin (BCG), additionally to surgical resection of the tumor. Last instillation was ten months before admission to our hospital. He had never suffered complications related to these installations besides to typical local reactions after each session (mild pain and low-grade fever).

At admission, he only complained about mild lower abdominal pain and a decrease in his urine rate. He neither had dysuria, urinary urgency nor hematuria. He did neither refer fever, dyspnea, cough or any other respiratory symptom. From the beginning, he was hemodynamically compromised, mainly with hypotension, requiring vasoactive drugs, suggesting septic shock.

First laboratory findings revealed lymphocytopenia with no leucopenia and marked elevation of acute phase reactants. As we were in a pandemic period, a nasopharyngeal swab specimen was taken and tested by reverse-transcription polymerase chain reaction (RT-PCR) for SARS-CoV-2, being positive. A lung computed tomography (CT) scan was performed, and no lung findings were observed. Serological test for SARSCoV-2 was also carried out with low levels of $\lg G$ and negative $\operatorname{lgM}$. An abdominal CT scan with contrast was also performed, with no new findings.

Three blood cultures for bacteria (aerobic and anaerobic) and a conventional urine culture were taken, being finally negative all of them. According to the unstable clinical situation of the patient, he was treated from the beginning with meropenem without clinical or analytical improvement (Figure 1).

Correspondence:

Pedro Manuel Garcia Ceberino, MD.

Internal Medicine Unit. Hospital Universitario Clínico San Cecilio.

Av/ Investigación s/n. (18016). Granada, Spain.

E-mail: pedrogarcebe@hotmail.com
BCG-related sepsis was a hypothesis and a Xpert ${ }^{\circledR}$ MTB/ RIF Ultra (Cepheid ${ }^{\circledR}$ ) was performed in a urine sample from the urinary bladder yielding a positive result (rifampicin resistance was not detected). Isoniazid, Rifampin and ethambutol were added to the combination treatment along with linezolid and corticosteroid pulses. Pyrazinamide was not added because intrinsic Mycobacterium bovis resistance. Thereafter, blood and urine cultures for mycobacteria were also taken, and a bone marrow puncture was performed, with any particular cell count finding nor granuloma.

Inflammatory parameters decreased 48 hours after initiation of antitiuberculous drugs. However, the patient suffered a myocardial infarction, malignant arrhythmia and sudden finally passed away. Blood, urine and bone narrow cultures for mycobacteria finally yielded a negative result.

Adjuvant intravesical therapy with bacillus Calmette-Guérin (BCG) attenuated strain of $M$. bovis instillations is a well-established therapy after transurethral resection [1]. It is a well-tolerated therapy, with low morbidity and a variable rate of side effects. Mild dysuria and urinary urgency and frequency are quite common soon after instillations, and until $24 \%$ of patients develop low grade fevers, general weakness and chills, without any confirmed infection $[2,3]$. Sometimes, mild prostatitis or orchitis have been documented, and could appear few months later [2].

BCG-related complications have different proposed mechanisms [2]. First of them, direct tissue injury (like in mycobacterial prostatitis and orchitis), or even systemic mycobacterial spread (mycobacterial or miliary pneumonitis, sepsis, etc). In those cases, mycobacteria can be cultured from blood, urine or liver biopsies [4]. It has also been hypothesized a systemic hypersensitivity reaction induced by immunologic stimulation [5], with uncommon mycobacterial isolation but with the presence of granulomas in histological samples.

On the other hand, major side effects, which take place in less than 5\% of patients, include lung and hepatic involve- 


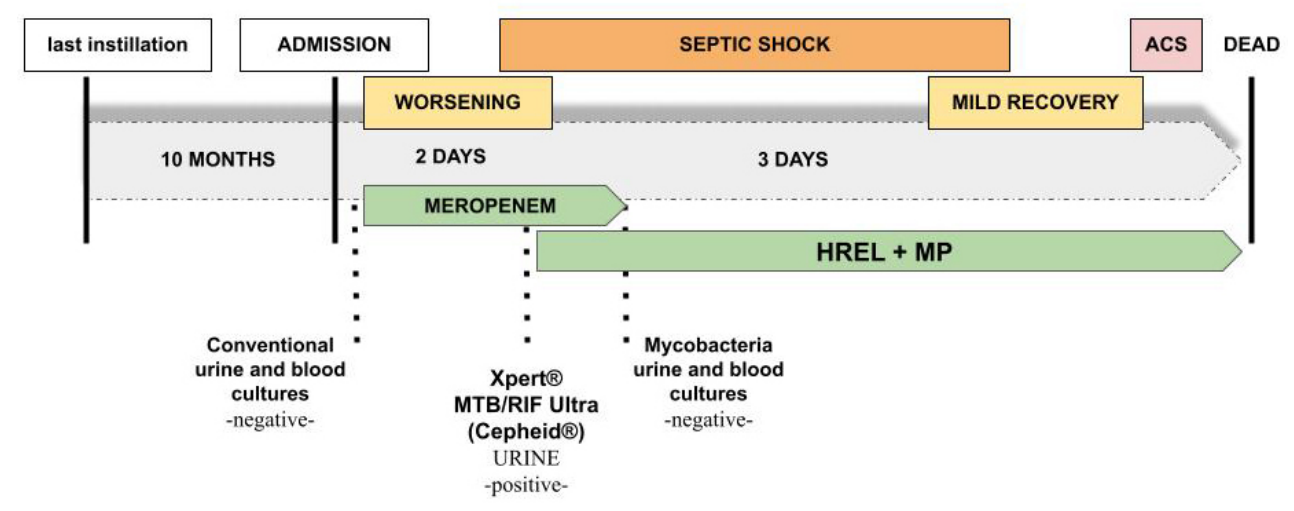

\begin{tabular}{l|l} 
Figure 1 & Timeline of cultures and clinical events.
\end{tabular}

HREL - Isoniazid, Rifampin, Ethambutol, Linezolid. MP - Corticosteroids pulses. ACS - Acute Coronary Syndrome.

ment, either by direct mycobacterial spread, or hypersensibility induced $[2,3]$. BCG-sepsis is pretty uncommon, being documented in only $0.3 \%$ of cases $[5,6]$. These complications can develop soon after, or even days or months after instillation $[2,3]$.

We could not get neither a positive blood nor a positive urine culture. Isolation of mycobacteria have always been tough, moreover if there is any specific organ to be sampled. Culture positivity rate is between $30-55.6 \%$, depending on organ involved or presentation [2,3]. Sensitivity of blood mycobacterial cultures is lower in severe sepsis and mycobacteremia can be fluctuating [5], moreover, if cultures for mycobacteria were taken after antituberculous treatment was initiated, as in our case.

Mycobacterium tuberculosis Xpert ${ }^{\circledR}$ MTB/RIF Ultra (Cepheid $\left.{ }^{\circledR}\right)$ in urine was positive; it is to say, a mycobacteria DNA isolation in urine. Detect M. bovis in urine has low positive predictive value weeks after last instillation until it washed out $[1,2]$. However, detection of leftovers of mycobacteria in urine is rare ten months after last instillation [7].

It has been documented the potential impact of immunosuppression (mainly steroid use, HIV, lymphoproliferative disorders and hypogammaglobulinemia) on the occurrence of BCG infection [8]. Reviewing literature, there is higher prevalence among patients with a long or intense immunosuppressive condition, but it have been not a headstone condition for developing severe forms $[1,8]$.

It could hypothesize a physiopathological relation between Iymphopenia induced by SARS-CoV-2 infection and reactivation of a latent $M$. bovis in urine tract.

In our report, SARS-CoV-2 infection without pneumonia and short lymphopenia must not have particular contribution for triggering BCGitis [8].

Furthermore, it is not found any relationship between SARS-CoV-2 pandemic scenario and an increment of mycobacteria reactivation rates [9].
The exclusion of other entities with any other microbiological finding (with appropriate samples extractions), and the absence of clinical and analytical improvement until antituberculosis treatment was initiated (with prompt response), along with the evidence of mycobacteria with DNA-assays in urine several months later, should guide the diagnosis of BCG-related sepsis $[1,2]$.

\section{FUNDING}

None to declare

\section{CONFLICTS OF INTEREST}

The authors declare no conflict of interest.

\section{REFERENCES}

1. Pérez-Jacoiste Asín MA, Fernández-Ruiz $M$, López-Medrano $F$, et al. Bacillus Calmette-Guérin (BCG) infection following intravesical BCG administration as adjunctive therapy for bladder cancer: incidence, risk factors, and outcome in a single-institution series and review of the literature. Medicine (Baltimore). 2014;93(17):236254. doi:10.1097/MD.0000000000000119

2. Cabas $P$, Rizzo $M$, Giuffrè $M$, et al. BCG infection (BCGitis) following intravesical instillation for bladder cancer and time interval between treatment and presentation: a systematic review. Urol Oncol 2021;39(2):85-92. doi:10.1016/j.urolonc.2020.11.037

3. Gonzalez OY, Musher DM, Brar I, et al. Spectrum of bacille Calmette-Guérin (BCG) infection after intravesical BCG immunotherapy. Clin Infect Dis. 2003;36(2):140-148. doi:10.1086/344908

4. Garmilla-Ezquerra P, Martínez-De Las Cuevas G, Hernández-Hernández JL. Granulomatous hepatitis and sepsis secondary to intravesical instillation of bacillus Calmette-Guérin. 2012;30(10): 656-657. doi:10.1016/j.eimc.2012.04.010 
5. Moreira E, Goleniuk D, Albornoz H. Disfunción orgánica múltiple como complicación de la instilación intravesical de bacilo de Calmette-Guérin. Comunicación de un caso y revisión de la literatura. Rev Méd Urug 2017; 33(4):261-268. doi: 10.29193/RMU.34.3.4

6. Elkabani M, Greene JN, Vincent AL, et al. Disseminated Mycobacterium bovis after intravesicular bacillus calmette-Gu rin treatments for bladder cancer. Cancer Control. 2000;7(5):476-481. doi:10.1177/107327480000700512

7. Van der Meijden AP. Practical approaches to the prevention and treatment of adverse reactions to BCG. Eur Urol. 1995;27(suppl1):23-28.

8. Yossepowitch O, Eggener S, Bochner BH, et al. Safety and efficacy of intravesical bacillus Calmette-Guerin Instillations in steroid treated and immunocompromised patients. J Urol. 2006;176:482485. doi.org/10.1016/j.juro.2006.03.066

9. Desouky, E. BCG versus COVID-19: impact on urology. World J Urol. 2021. 39,823-827. doi.org/10.1007/s00345-020-03251-7 